\title{
Vanishing of mode $B$ in the wake behind a rotationally oscillating circular cylinder
}

\author{
Philippe Poncet ${ }^{\mathrm{a})}$ \\ LMC-IMAG, Université Joseph Fourier, B.P. 53, 38041 Grenoble Cedex 09, France
}

(Received 10 December 2001; accepted 27 March 2002; published 6 May 2002)

\begin{abstract}
This Brief Communication presents a numerical investigation of the dimensionality of a flow behind a three-dimensional rotationally oscillating circular cylinder. These computations are performed at a Reynolds number $\mathrm{Re}=500$, which is at a level where the mode $\mathrm{B}$ is the dominant three-dimensional instability. We show that a rotation of high enough amplitude makes the 3D instabilities vanish and brings the flow back to its nominal two-dimensional state. (C) 2002 American Institute of Physics. [DOI: 10.1063/1.1479344]
\end{abstract}

The von Kármán alleys generated in the wake of a circular cylinder are, at low Reynolds numbers, a time-periodic and two-dimensional flow. It is now well known from both experiments ${ }^{1,2}$ and numerical investigations ${ }^{3,4}$ that this nominally two-dimensional flow becomes three-dimensional when $\operatorname{Re}>190$.

The three-dimensional structures of vorticity are present into two forms, called mode $\mathrm{A}$ and mode $\mathrm{B}$, with wavelength of $4 D$ and $0.8 D$, respectively, $D$ being the diameter of the cylinder. These two three-dimensional modes are the only stable modes, at least up to $\operatorname{Re}=1900,{ }^{5}$ though the wavelength of mode A tends to decrease as the Reynolds number increases. ${ }^{6}$ Vortex dislocation may occur by means of interaction between mode $\mathrm{A}$ and mode $\mathrm{B} .^{2}$ Moreover, mode B structures are clearly orthogonal to the axis of the cylinder and to the von Kármán alleys. While the Reynolds number increases, there is a gradual transfer of energy from mode A to mode $\mathrm{B}$, and at $\mathrm{Re}=500$, chosen for our simulations, mode $\mathrm{B}$ is considered as the dominant three-dimensional effect.

This Brief Communication focuses on the behavior of mode B instabilities when the cylinder is rotationally oscillating. In the bidimensional case, it has been shown that a rotationally oscillating cylinder can lead to different kinds of von Kármán alleys in its wake, with various strengths and frequencies depending on the rotation parameters. ${ }^{7}$ The present work aims at explaining how the threedimensionality of the flow interacts with these alleys.

Frequencies are chosen at once and twice the Strouhal number, corresponding to natural and the first superharmonic of the vortex shedding. For both these frequencies, we show that the flow turns back to its two-dimensional configuration when the amplitude is high enough (half a revolution in our case).

The computational approach is summarized as follows. We perform direct numerical simulations of the NavierStokes equations in their velocity-vorticity formulation:

${ }^{\text {a)} E l e c t r o n i c ~ m a i l: ~ p h i l i p p e . p o n c e t @ i m a g . f r ~}$

$$
\frac{\partial \boldsymbol{\omega}}{\partial t}+\mathbf{u} \cdot \boldsymbol{\nabla} \boldsymbol{\omega}-\boldsymbol{\omega} \cdot \boldsymbol{\nabla} \mathbf{u}-\nu \nabla^{2} \boldsymbol{\omega}=0
$$

over a cylindrical domain $\Omega$. The computational domain is an "O-grid," whose spanwise length is $0 \leqslant z \leqslant L=\pi D$, and radius in $1 \leqslant r \leqslant 1+4 \pi$. The spanwise length is not critical for our investigation since we are focusing our attention on mode B. The Reynolds number is defined as $\operatorname{Re}=U_{\infty} D / \nu$, where $D$ is the diameter of the cylinder, $U_{\infty}$ the free stream velocity, and $\nu$ the kinematic viscosity.

The numerical method is a particle in cell method (hybrid vortex method), in the spirit of Cottet and Koumoutsakos. ${ }^{8}$ The boundary layer is computed by a flux of vorticity on the cylinder. ${ }^{9}$ The three parts of the NavierStokes equations, i.e., the convection, the diffusion, and the flux of vorticity, are successively solved in a fractional-step algorithm. ${ }^{8-10}$ A periodic third-order remeshing, critical for accuracy, is performed in order to maintain the regularity of the particle locations. Several simulation of 3D unbounded flows have been performed in order to validate the numerical method. ${ }^{11,12}$

The simulation begins with a slight rotation in order to trigger the $2 \mathrm{D}$ von Kármán instability, when $t<4$. Then we let the three-dimensionality grow until it reaches saturation. The mode B wavelength being $0.8 D{ }^{3}$ we get four couples of contrarotating fingers of vorticity, i.e., eight vortex structures, in the computational domain. Two interesting features of the flow, in order to measure the three-dimensionality, are the spanwise enstrophy,

$$
\left\|\omega_{z}\right\|_{2}^{2}=\int_{\Omega} \omega_{z}(\xi)^{2} d \xi,
$$

and the orthogonal enstrophy,

$$
\left\|\omega_{x}\right\|_{2}^{2}+\left\|\omega_{y}\right\|_{2}^{2}=\int_{\Omega} \omega_{x}(\xi)^{2}+\omega_{y}(\xi)^{2} d \xi
$$

which extracts the enstrophy contained in the crosswise and streamwise vortex structures from the whole enstrophy.

The nominal drag (when the flow is still bidimensional) of the flow past a nonrotating cylinder is 1.45 and close to 


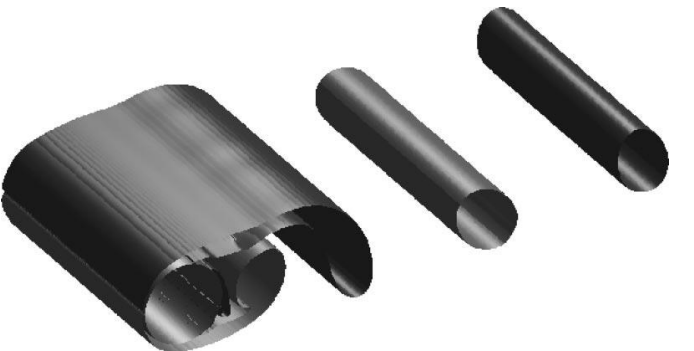

FIG. 1. Surface of isovorticity of the nominal 2D state.

Henderson's benchmark value of $1.445 .^{13}$ Threedimensionality makes the drag coefficient decrease down to 1.2 , which can be compared to the value of 1.24 provided by Mittal and Balachandar ${ }^{14}$ at $\mathrm{Re}=525$, though both are overestimated, in the present case because of the spanwise short length of the cylinder. Indeed, simulations with larger nonrotating cylinders give 1.17 , which is the same as Wieselsberger's. ${ }^{15}$ The present nominal Strouhal number is found at $S_{t}=0.229$ and also close to Henderson's, at $0.228 .{ }^{13}$ The present $3 D$ Strouhal number is 0.220 , once again close to Mittal and Balachandar's, ${ }^{14}$ i.e., 0.222 , though both are higher than experimental Strouhal numbers. Present wavelength of the mode $\mathrm{B}$ is $0.8 \mathrm{D}$ while Barkley and Henderson ${ }^{3}$ predict this wavelength at $0.82 \mathrm{D}$, with similar spectral profiles, which ensures that mode B instabilities are correctly computed in the present simulations.

Once the three-dimensionality is fully developed, in our case at $t_{c}=110$, the cylinder begins to rotate, with a tangential velocity on the body given by

$$
V_{\text {body }}=A \pi S_{f} R \sin \left(\pi S_{f} t\right),
$$

where $S_{f}$ is the forced Strouhal number and $A$ is the amplitude of rotation.

Figures 1 and 2 show the nominally bidimensional surface of isovorticity, and this surface once the mode $B$ is developed. We consider only $S_{f}$ as a multiple of $S_{t}$, the natural Strouhal number.

When a high-amplitude rotation is performed, half a circumference in our case, we see that strong alleys are created. These alleys are strong enough to unhook the fingers of vorticity from the cylinder: as a result, these fingers are brought away from the cylinder, and the flow becomes laminar and bidimensional in a growing neighborhood of the cylinder.

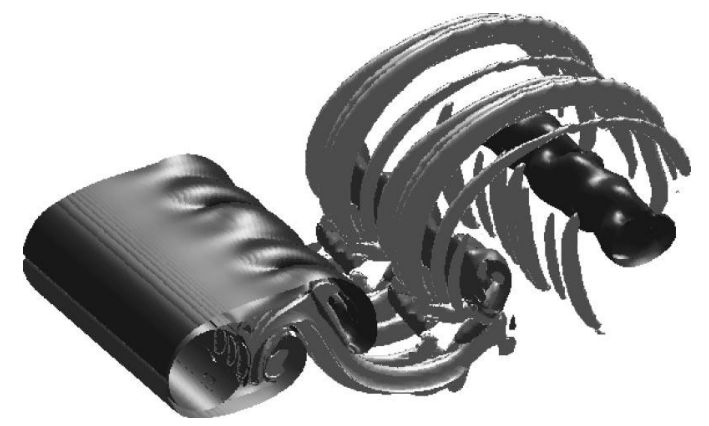

FIG. 2. Surface of isovorticity when the 3D instabilities are fully developed.
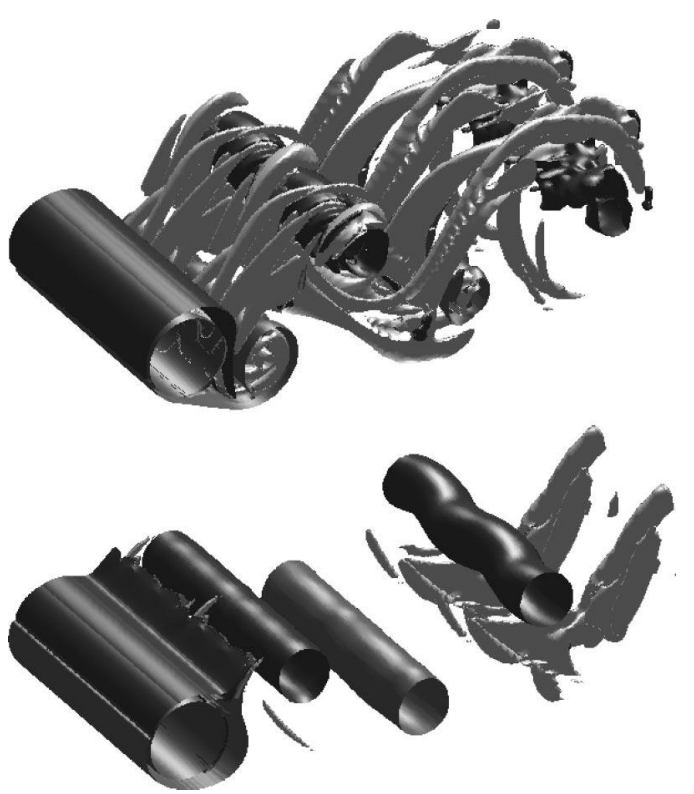

FIG. 3. Surfaces of isovorticity after activation of the rotation with $S_{f}$ $=S_{t}$. Above: $t=120$, below: $t=135$. Rotation starts at $t=110$.

This mechanism, in the case $S_{f}=S_{t}$, i.e., when the rotation frequency is locked on the natural vortex shedding, is exhibited on Fig. 3. We see that for a while, at $t=120=t_{c}$ +10 , the flow is still fully three-dimensional, and even more than at $t=t_{c}$. But after a few shedding cycles the instabilities are sent downstream. After a few periods, no visible instability remains. Nevertheless, a very small amount $[0.4 \%$ of the initial orthogonal enstrophy, see Eq. (3)] of threedimensionality remains in the flow. We consider this residual amount as insignificant.

Furthermore, the case $A=\pi / 3$ has been also computed, and does not lead to a bidimensional state (see Fig. 4). Indeed, the orthogonal enstrophy tends (roughly) to 80, which represents more than half its value at $t=t_{c}$, that is to say, a three-dimensional state. This also shows the possibility of a bifurcation between $\pi / 3$ and $\pi / 2$.

One also considers the rotation frequency as the first super-harmonic, i.e., $S_{f}=2 S_{t}$. In this case, the instabilities also vanish. Indeed, we see in Fig. 5 that instabilities are brought away as well as if $S_{f}=S_{t}$. For this high-frequency

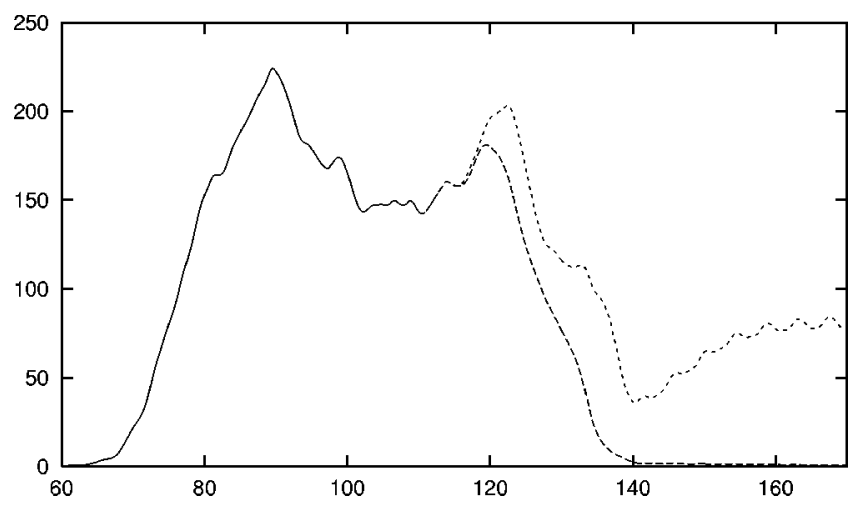

FIG. 4. Orthogonal enstrophy $\left\|\omega_{x}\right\|_{2}^{2}+\left\|\omega_{y}\right\|_{2}^{2}$ versus time for a rotation with $S_{f}=S_{t} .---: A=\pi / 2,---: A=\pi / 3$. 

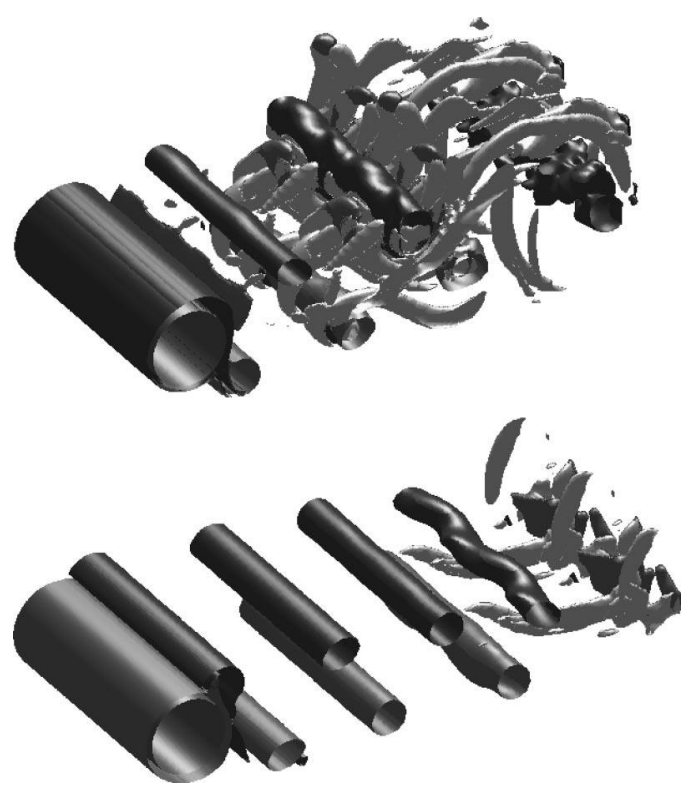

FIG. 5. Isovorticity surfaces after activation of the rotation with $S_{f}=2 S_{t}$. Above: $t=120$, below: $t=130$. Rotation starts at $t=110$.

rotation, the orthogonal enstrophy (3) decreases down to $10^{-6}$, which means that three-dimensionality vanishes completely.

The vanishing of mode B is shown on Fig. 6, on which the orthogonal enstrophy $\left\|\omega_{x}\right\|_{2}^{2}+\left\|\omega_{y}\right\|_{2}^{2}$ is plotted for both $S_{f}=S_{t}$ and $S_{f}=2 S_{t}$. As we have already mentioned, this quantity measures the amount of vorticity orthogonal to the axis of the cylinder, that is to say, the streamwise and crosswise vorticity. These curves, on Fig. 6 , show that at $t=140$ $=t_{c}+30$ the flow is bidimensional in the region defined by

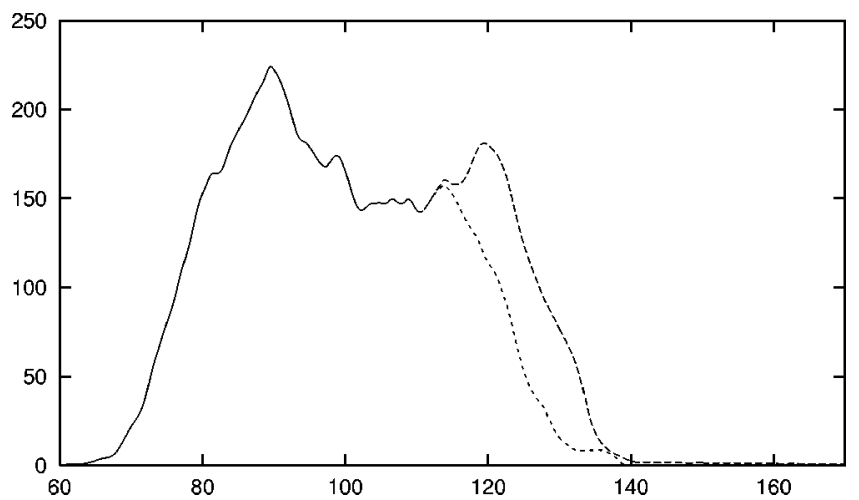

FIG. 6. Orthogonal enstrophy $\left\|\omega_{x}\right\|_{2}^{2}+\left\|\omega_{y}\right\|_{2}^{2}$ versus time for a rotation with $A=\pi / 2$. ---: $S_{f}=S_{t}$, ---: $S_{f}=2 S_{t}$. the computational domain. Moreover, Figs. 3 and 5 exhibit snapshots of the isovorticity surface when three-dimensional structures have not yet vanished.

As a conclusion, we have shown numerically that the nonlinear three-dimensional flow created past a circular cylinder can be laminarized by means of a rotation of this cylinder. This has been shown in the case of a rotation of half a revolution and at two values of frequency.

This behavior might be valid only in a neighborhood of the cylinder, even if it is large compared to the radius. Indeed, the rotation gives strength to the von Kármán alleys and once these alleys fade, by means of the diffusion, there might be a critical distance after which three-dimensional structures are stable. In order to elucidate how this hypothetical critical distance depends on the rotation parameters, further, in-depth numerical investigations are required.

Moreover, the rotation plays certainly a critical role in the way back to a bidimensional state of the flow. Figure 4 shows that in the case $S_{f}=S_{t}$, the bifurcation amplitude is between $\pi / 3$ and $\pi / 2$. A systematic parametric study with a larger cylinder is under way in order to elucidate bifurcation patterns.

${ }^{1}$ M. Brede, H. Eckelman, and D. Rockwell, "On secondary vortices in the cylinder wake,” Phys. Fluids 8, 2117 (1996).

${ }^{2}$ C. H. K. Williamson, "Three-dimensional wake behind a cylinder," J. Fluid Mech. 328, 345 (1996).

${ }^{3}$ D. Barkley and R. D. Henderson, "Three-dimensional Floquet stability analysis of the wake of a circular cylinder," J. Fluid Mech. 322, 215 (1996).

${ }^{4}$ M. C. Thompson, K. Hourigan, and J. Sheridan, "Three-dimensional instabilities in the wake of a circular cylinder," Exp. Therm. Fluid Sci. 12, 190 (1996).

${ }^{5}$ M. F. Unal and D. Rockwell, "On vortex formation from a cylinder. Part I, the initial stability," J. Fluid Mech. 190, 491 (1988).

${ }^{6}$ C. H. K. Williamson, "Mode A secondary instability in wake transition," Phys. Fluids 8, 1680 (1996).

${ }^{7}$ S. C. R. Dennis, P. Nguyen, and S. Kocabiyik, "The flow induced by a rotationally oscillating and translating circular cylinder," J. Fluid Mech. 385, 255 (2000).

${ }^{8}$ G.-H. Cottet and P. D. Koumoutsakos, Vortex Methods, Theory and Practice (Cambridge University Press, Cambridge, 2000).

${ }^{9}$ P. D. Koumoutsakos and A. Leonard, "High-resolution simulations of the flow around an impulsively started cylinder using vortex methods," J. Fluid Mech. 296, 1 (1995).

${ }^{10}$ P. Poncet, Ph.D. thesis, Univ. Joseph Fourier, Grenoble, France, 2001.

${ }^{11}$ G.-H. Cottet, B. Michaux, S. Ossia, and G. Vanderlinden, "A comparison of spectral and vortex methods in three-dimensional incompressible flows," J. Comput. Phys. 175, 702 (2002).

${ }^{12}$ M. L. Ould Sahili, G.-H. Cottet, and M. El Hamraoui, "Blending finitedifference and vortex methods for incompressible flow computations," SIAM J. Sci. Comput. (USA) 22, 1655 (2000).

${ }^{13}$ R. D. Henderson, "Details of the drag curve near the onset of vortex shedding," Phys. Fluids 7, 2102 (1995).

${ }^{14}$ R. Mittal and S. Balachandar, "Effects of three-dimensionality on the lift and drag of nominally two-dimensional flows," Phys. Fluids 7, 1841 (1995).

${ }^{15} \mathrm{C}$. Wieselsberger, "New data on the laws of fluid resistance," NACA Tech. Rep. TN-84 (1922). 